\title{
POSITIVE SOLUTIONS OF INTEGRODIFFERENTIAL EQUATIONS ${ }^{1}$
}

\author{
CH. G. PHILOS \\ University of Ioannina \\ Department of Mathematics \\ P.O. Box 1186 \\ 45110 Ioannina, GREECE
}

\begin{abstract}
Integrodifferential equations of the forms $x^{\prime}(t)+p(t) \int_{0}^{t} K(t-s) x(s) d s=0$ and $x^{\prime}(t)+q(t) \int_{-\infty}^{t} K(t-s) x(s) d s=0$

are considered, where $K \in C([0, \infty),[0, \infty)), \quad p \in C([0, \infty),[0, \infty))$ and $q \in C((-\infty, \infty),[0, \infty))$. Necessary conditions and also sufficient conditions for the existence of positive solutions are established.
\end{abstract}

Key words: Positive solution, integrodifferential equation.

AMS (MOS) subject classifications: $\quad 34 \mathrm{~K} 15,34 \mathrm{C} 10$.

\section{INTRODUCTION AND STATEMENT OF RESULTS}

Our aim in this paper is to obtain necessary conditions and also sufficient conditions for the existence of positive solutions of certain integrodifferential equations. The literature is scarce concerning the oscillation and nonoscillation of solutions of integrodifferential equations. Only very recently Gopalsamy [3-8], Ladas, the present author and Sficas [9], the present author [10,11], and the present author and Sficas [12] studied the oscillatory behavior of solutions of integrodifferential equations or of systems of integrodifferential equations. For questions related to the theory of integrodifferential equations, see Burton [1]. Also, it is to be noted that integrodifferential equations can be regarded as differential equations with unbounded delays. See Corduneanu and Lakshmikantham [2] for a survey on equations with unbounded delays.

Consider the integrodifferential equations

$$
x^{\prime}(t)+p(t) \int_{0}^{t} K(t-s) x(s) d s=0
$$

and

\footnotetext{
${ }^{1}$ Received: January, 1992. Revised: October, 1992.
} 


$$
x^{\prime}(t)+q(t) \int_{-\infty}^{t} K(t-s) x(s) d s=0
$$

where $K$ is a nonnegative continuous function on the interval $[0, \infty), p$ is a nonnegative continuous function on $[0, \infty)$ and $q$ is a nonnegative continuous function on the real line $R$. Consider also the integrodifferential inequalities

and

$$
y^{\prime}(t)+p(t) \int_{0}^{t} K(t-s) y(s) d s \leq 0
$$

$$
y^{\prime}(t)+q(t) \int_{-\infty}^{t} K(t-s) y(s) d s \leq 0 .
$$

If $T \geq 0$, by a solution on $[T, \infty)$ of $\left(E_{1}\right)$ [resp. of $\left.\left(I_{1}\right)\right]$ we mean a continuous function $x$ [resp. $y$ ] on $[0, \infty)$, which is continuously differentiable on $[T, \infty)$ and satisfies $\left(E_{1}\right)$ [resp. $\left.\left(I_{1}\right)\right]$ for every $t \geq T$. If $T \in R$, then a solution on $[T, \infty)$ of $\left(E_{2}\right)$ [resp. of $\left.\left(I_{2}\right)\right]$ is a continuous function $x$ [resp. $y$ ] on the real line $R$, which is continuously differentiable on $[T, \infty)$ and satisfies $\left(E_{2}\right)$ [resp. $\left(I_{2}\right)$ ] for all $t \geq T$. Also, a continuously differentiable function $y$ on $R$, which satisfies $\left(I_{2}\right)$ for every $t \in R$, is called a solution on $R$ of $\left(I_{2}\right)$.

Necessary conditions are presented for $\left(E_{1}\right)$, or more generally for $\left(I_{1}\right)$, to have solutions on $[T, \infty)$, where $T \geq 0$, which are positive on $[0, \infty)$. Analogously, necessary conditions are given for $\left(E_{2}\right)$, or more generally for $\left(I_{2}\right)$, to have solutions on $[T, \infty)$, where $T \in R$, which are positive on $R$. On the other hand, sufficient conditions are obtained for $\left(E_{1}\right)$ to have a solution on $[T, \infty)$ where $T>0$, which is positive on $[0, \infty)$ and tends to zero at $\infty$. Similarly, sufficient conditions are derived for the existence of a solution on $[T, \infty)$ of $\left(E_{2}\right)$, where $T \in R$, which is positive on $R$ and tends to zero at $\infty$.

More precisely, in this paper the following results are proved.

Theorem 1: $\quad$ Suppose that there exists a $\tau_{0}>0$ such that $K$ is not identically zero on $\left[\tau_{0}, \infty\right)$ and

Moreover, assume that

$$
\liminf _{t \rightarrow \infty}^{t+\tau_{0}} \int_{t}^{t} p(s) d s>0
$$

$$
-\lambda+\sup _{t \geq 0} \int_{0}^{t} K(s) e^{\lambda \int^{t}-s\left[\operatorname{sinf}_{\left.\xi \geq u^{p(\xi)}\right] d u}^{d u}\right.} d s>0 \text { for all } \lambda>0 .
$$

Let $T \geq 0$. Then there is no solution on $[T, \infty)$ of $\left(I_{1}\right)\left[\right.$ and in particular of $\left.\left(E_{1}\right)\right]$ which is 
positive on $[0, \infty)$.

Theorem 2: $\quad$ Suppose that there exists a $\tau_{0}>0$ such that $K$ is not identically zero on $\left[\tau_{0}, \infty\right)$ and

Moreover, assume that

$$
\liminf _{t \rightarrow \infty}^{t+\tau_{0}} \int_{t}^{t(s) d s>0}
$$

$$
-\lambda+\sup _{t \geq 0} \int_{0}^{t} K(s) e^{\lambda \int_{t}^{t}\left[\operatorname{sinf}_{\xi} q(\xi) u^{(\xi)}\right] d u} d s>0 \text { for all } \lambda>0 .
$$

Let $T \in R$. Then there is no solution on $[T, \infty)$ of $\left(I_{2}\right)\left[\right.$ and in particular of $\left.\left(E_{2}\right)\right]$ which is positive on $R$.

Theorem 3: $\quad$ Let $y$ be a positive solution on $[0, \infty)$ of $\left(I_{1}\right)$. Moreover, let $T>0$ and suppose that $K$ is not identically zero on $[0, T]$ and $p$ is positive on $[T, \infty)$. Then there exists a solution $x$ on $[T, \infty)$ of $\left(E_{1}\right)$, which is positive on $[0, \infty)$ and such that

$$
\begin{gathered}
x(t) \leq y(t) \text { for every } t \geq 0, \\
\lim _{t \rightarrow \infty} x(t)=0
\end{gathered}
$$

and

$$
x^{\prime}(t)+p(t) \int_{0}^{t} K(t-s) x(s) d s \leq 0 \text { for } 0 \leq t<T
$$

Corollary 1: $\quad$ Let the following hypothesis be satisfied:

$$
-\lambda+\sup _{t \geq 0} \int_{0}^{t} K(s) e^{\lambda \int^{t} p(u) d u} d s \leq 0 \text { for some } \lambda>0 .
$$

Moreover, let $T>0$ and suppose that $K$ is not identically zero on $[0, T]$ and $p$ is positive on $[T, \infty)$. Then there exists a solution $x$ on $[T, \infty)$ of $\left(E_{1}\right)$, which is positive on $[0, \infty)$ and satisfies (1), (2) and

$$
x(t) \leq e^{-\lambda \int_{0}^{t} p(u) d u} \text { for every } t \geq 0
$$

Theorem 4: $\quad$ Assume that $K$ is not identically zero on $[0, \infty)$. Let $y$ be a positive solution on $R$ of $\left(I_{2}\right)$. Moreover, let $T \in R$ and suppose that $q$ is positive on $[T, \infty)$. Then there exists a solution $x$ on $[T, \infty)$ of $\left(E_{2}\right)$, which is positive on $R$ and such that (1) holds and 


$$
\begin{gathered}
x(t) \leq y(t) \text { for every } t \in R, \\
x^{\prime}(t)+q(t) \int_{-\infty}^{t} K(t-s) x(s) d s \leq 0 \text { for } t<T .
\end{gathered}
$$

Corollary 2: $\quad$ Assume that $K$ is not identically zero on $[0, \infty)$. Let the following hypothesis be satisfied:

$$
-\lambda+\sup _{t \in R} \int_{0}^{\infty} K(s) e^{\lambda \int^{t} q(u) d u} d s \leq 0 \text { for some } \lambda>0 .
$$

Moreover, let $T \in R$ and suppose that $q$ is positive on $[T, \infty)$. Then there exists a solution $x$ on $[T, \infty)$ of $\left(E_{2}\right)$, which is positive on $R$ and satisfies (1), (3) and

$$
x(t) \leq e^{-\lambda \int_{0}^{t} q(u) d u} \text { for every } t \in R .
$$

In the special case where $p(t)=1, t \geq 0$ and $q(t)=1, t \in R$ our results lead to previous known ones, see Ladas, Philos and Sficas [9] and Philos [11]. In this special case, a combination of Theorem 1 and Corollary 1 leads to a necessary and sufficient condition for $\left(E_{1}\right)$ to have solutions on $[T, \infty)$, where $T>0$, which are positive on $[0, \infty)$; analogously, by combining Theorem 2 and Corollary 2, a necessary and sufficient condition can be obtained for the existence of a solution on $[T, \infty)$ of $\left(E_{2}\right)$, where $T \in R$, which is positive on $R$.

The results of the present paper can be extended to the more general case of integrodifferential equations (and inequalities), which involve the term $\sum_{n=0}^{\infty} \mu_{n} x\left(t-\tau_{n}\right.$ ) where $\mu_{n}$ and $\tau_{n}$ are nonnegative constants for $n=0,1, \ldots$ and $\sup \left\{\tau_{n}: n=0,1, \ldots\right\}<\infty$. Such an extension was recently presented in [11] for the special case where $p(t)=1, t \geq 0$ and $q(t)=1$, $t \in R$. Also, our results can be extended to systems of integrodifferential equations (and inequalities); see [10] for such an extension in a particular case.

\section{PROOFS OF THE RESULTS}

Theorem 2 is essentially a consequence of Theorem 1 . Corollary 1 follows from Theorem 3 and Corollary 2 is obtained from Theorem 4.

Proof of Theorem 1: Assume, for the sake of contradiction, that $\left(I_{1}\right)$ has a solution $y$ on $[T, \infty)$ which is positive on $[0, \infty)$. From $\left(I_{1}\right)$ it follows immediately that $y^{\prime}(t) \leq 0$ for every $t \geq T$ and so $y$ is decreasing on the interval $[T, \infty)$. 


\section{Define}

$$
\Lambda=\left\{\lambda>0: y^{\prime}(t)+\lambda p(t) y(t) \leq 0 \text { for all large } t\right\}
$$

The set $\Lambda$ is nonempty. In fact, condition $\left(C_{1}\right)$ implies that $K$ is not identically zero on the interval $[0, \infty)$ and hence we can choose a $\tau^{*}>0$ such that

$$
\lambda_{0} \equiv \int_{0}^{\tau_{0}^{*}} K(s) d s>0 .
$$

Then, by using the decreasing character of $y$ on $[T, \infty)$, from $\left(I_{1}\right)$ we obtain for every $t \geq T+\tau^{*}$

$$
\begin{gathered}
0 \geq y^{\prime}(t)+p(t) \int_{0}^{t} K(t-s) y(s) d s=y^{\prime}(t)+p(t) \int_{0}^{t} K(s) y(t-s) d s \\
\geq y^{\prime}(t)+p(t) \int_{0}^{t-T} K(s) y(t-s) d s \geq y^{\prime}(t)+p(t)\left[\int_{0}^{t-T} K(s) d s\right] y(t) \\
\geq y^{\prime}(t)+p(t)\left[\int_{0}^{\tau^{*}} K(s) d s\right] y(t)=y^{\prime}(t)+\lambda_{0} p(t) y(t) .
\end{gathered}
$$

This means that $\lambda_{0} \in \Lambda$ and consequently, $\Lambda \neq \emptyset$. It is clear that the set $\Lambda$ is a subinterval of $(0, \infty)$ such that inf $\Lambda=0$.

Next, we will show that $\Lambda$ is bounded from above. Since $K$ is not identically zero on the interval $\left[\tau_{0}, \infty\right)$, there exist $\tau_{1}, \tau_{2}$ with $\tau_{0}<\tau_{1}<\tau_{2}$ such that

$$
A \equiv \int_{\tau_{1}}^{\tau_{2}} K(s) d s>0
$$

By the decreasing nature of $y$ on $[T, \infty)$, it follows from $\left(I_{1}\right)$ that for $t \geq T+\tau_{2}$

$$
\begin{gathered}
0 \geq y^{\prime}(t)+p(t) \int_{0}^{t} K(t-s) y(s) d s \geq y^{\prime}(t)+p(t) \int_{T}^{t-\tau_{1}} K(t-s) y(s) d s \\
\geq y^{\prime}(t)+p(t)\left[\int_{T}^{t-\tau_{1}} K(t-s) d s\right] y\left(t-\tau_{1}\right)=y^{\prime}(t)+p(t)\left[\int_{\tau_{1}}^{t-T} K(s) d s\right] y\left(t-\tau_{1}\right)
\end{gathered}
$$


That is,

$$
\geq y^{\prime}(t)+p(t)\left[\int_{\tau_{1}}^{\tau_{2}} K(s) d s\right] y\left(t-\tau_{1}\right)
$$

$$
y^{\prime}(t)+A p(t) y\left(t-\tau_{1}\right) \leq 0 \text { for all } t \geq T+\tau_{2} .
$$

Thus, by taking into account the fact that $y$ is decreasing on $[T, \infty)$, we obtain for every $t \geq T+\tau_{2}$

$$
\begin{aligned}
y(t) \geq y\left(t+\tau_{0}\right) & +A \int_{t}^{t+\tau_{0}} p(s) y\left(s-\tau_{1}\right) d s>A \int_{t}^{t+\tau_{0}} p(s) y\left(s-\tau_{1}\right) d s \\
\geq A & {\left[\int_{t}^{t+\tau_{0}} p(s) d s\right] y\left(t+\tau_{0}-\tau_{1}\right) . }
\end{aligned}
$$

But, condition ( $i$ ) guarantees that there exist a positive constant $B$ and a $T^{*} \geq T+\tau_{2}$ such that

Therefore,

$$
\int_{t}^{t+\tau_{0}} p(s) d s \geq B \text { for all } t \geq T^{*}
$$

$$
y(t)>L_{0} y\left(t-\left(\tau_{1}-\tau_{0}\right)\right) \text { for every } t \geq T^{*},
$$

where $L_{0}=A B>0$. Since $y$ is decreasing on $[T, \infty)$, we always have $L_{0}<1$. Let us consider a positive integer $m$ such that $m\left(\tau_{1}-\tau_{0}\right) \geq \tau_{0}$. Then, by (5), we obtain for $t \geq \tilde{T} \equiv T^{*}+$ $(m-1)\left(\tau_{1}-\tau_{0}\right)$

$$
y(t)>L_{0} y\left(t-\left(\tau_{1}-\tau_{0}\right)\right)>L_{0}^{2} y\left(t-2\left(\tau_{1}-\tau_{0}\right)\right)>\ldots>L_{0}^{m} y\left(t-m\left(\tau_{1}-\tau_{0}\right)\right) .
$$

So, by the decreasing nature of $y$ on $[T, \infty)$, it follows that

$$
y(t)>L y\left(t-\tau_{0}\right) \text { for all } t \geq \tilde{T},
$$

where $L=L_{0}^{m}, 0<L<1$. We now claim that $\vartheta \equiv-\frac{1}{B} \ell n L>0$ is an upper bound of $\Lambda$. Otherwise, $\vartheta \in \Lambda$ and hence there exists a $T_{\vartheta} \geq T$ such that

$$
y^{\prime}(t)+\vartheta p(t) y(t) \leq 0 \text { for every } t \geq T_{\vartheta}
$$

So, if we set

$$
\phi_{\vartheta}(t)=y(t) e^{\vartheta \int_{\vartheta}^{t} p(s) d s}, t \geq T_{\vartheta}
$$

then we have for $t \geq T_{\vartheta}$ 


$$
\phi_{\vartheta}^{\prime}(t)=\left[y^{\prime}(t)+\vartheta p(t) y(t)\right] e^{\vartheta \int_{T}^{t} p(s) d s} \leq 0
$$

which means that $\phi_{\vartheta}$ is decreasing on $\left[T_{\vartheta}, \infty\right)$. Thus, for every $t \geq T_{\vartheta}+\tau_{0}$

$$
y(t) e^{\vartheta \int_{T_{\vartheta}}^{t} p(s) d s} \equiv \phi_{\vartheta}(t) \leq \phi_{\vartheta}\left(t-\tau_{0}\right) \equiv y\left(t-\tau_{0}\right) e^{\vartheta \int_{\vartheta}^{t-\tau_{0}} p(s) d s}
$$

and consequently

$$
y(t) \leq y\left(t-\tau_{0}\right) e^{-\vartheta \int^{t} p(s) d s} \text { for all } t \geq T_{\vartheta}+\tau_{0}
$$

Hence, by using (4), we obtain for $t \geq \widehat{T}_{\vartheta} \equiv \max \left\{T_{\vartheta}+\tau_{0}, T^{*}+\tau_{0}\right\}$

$$
y(t) \leq y\left(t-\tau_{0}\right) e^{-\vartheta \int_{t-\tau_{0}}^{\left(t-\tau_{0}\right)+\tau_{0}} p(s) d s} \leq y\left(t-\tau_{0}\right) e^{-\vartheta B} .
$$

That is,

$$
y(t) \leq L y\left(t-\tau_{0}\right) \text { for every } t \geq \widehat{T}_{\vartheta} .
$$

This contradicts (6) and the proof of the claim is complete.

Now, we put $\lambda^{*}=\sup \Lambda$ and we consider an arbitrary number $\mu$ with $0<\mu<\lambda^{*}$. Then $r \equiv \lambda^{*}-\mu \in\left(0, \lambda^{*}\right)$ and so $r$ is an element of $\Lambda$. Thus, there exists a $T_{r} \geq T$ such that

$$
y^{\prime}(t)+r p(t) y(t) \leq 0 \text { for all } t \geq T_{r} .
$$

For any $t, s$ with $t \geq T_{r}$ and $0 \leq s \leq t-T_{r}$, we get

$$
\frac{y(t-s)}{y(t)}=e^{-\ln \frac{y(t)}{y(t-s)}}=e^{-\int_{t-s}^{t} \frac{y^{\prime}(u)}{y(u)} d u} \geq e^{r \int_{t-s}^{t} p(u) d u} .
$$

That is,

$$
y(t-s) \geq y(t) e^{r \int_{t-s}^{t} p(u) d u} \text { for } t \geq T_{r} \text { and } 0 \leq s \leq t-T_{r} .
$$

But, $\left(I_{1}\right)$ gives for $t \geq T_{r}$

$$
0 \geq y^{\prime}(t)+p(t) \int_{0}^{t} K(s) y(t-s) d s \geq y^{\prime}(t)+p(t) \int_{0}^{t-T_{r}} K(s) y(t-s) d s .
$$

So, by using (7), we obtain

$$
y^{\prime}(t)+\left[\int_{0}^{t-T_{r}} K(s) e^{r \int_{t-s}^{t} p(u) d u} d s\right] p(t) y(t) \leq 0 \text { for } t \geq T_{r}
$$


Let us suppose that there exists a $v \geq T_{r}$ such that

$$
\inf _{t \geq v}^{t-T_{r}} K(s) e^{r \int_{t-s}^{t} p(u) d u} d s \equiv \hat{\lambda}>\lambda^{*}
$$

Then, in view of (8), we have

$$
y^{\prime}(t)+\hat{\lambda} p(t) y(t) \leq 0 \text { for every } t \geq v
$$

and consequently $\hat{\lambda} \in \Lambda$. This is impossible, since $\hat{\lambda}>\lambda^{*} \equiv \sup \Lambda$. Thus, we must have

This gives

$$
i_{t \geq v}^{t-T_{r}} \int_{0}^{r} K(s) e^{r \int_{t-s}^{p(u) d u}} d s \leq \lambda^{*} \text { for all } v \geq T_{r} \text {. }
$$

that is

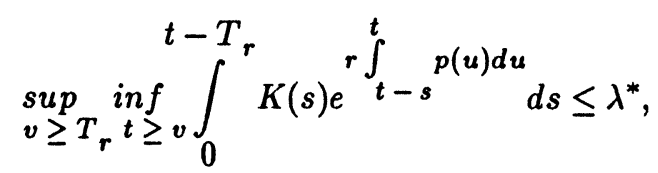

Therefore,

$$
\liminf _{t \rightarrow \infty} \int_{0}^{t-T_{r}} K(s) e^{r \int_{t-s}^{t} p(u) d u} d s \leq \lambda^{*}
$$

So, if we define

$$
\lim _{t \rightarrow \infty} \inf \int_{0}^{t} K(s) e^{r \int_{t-s}^{t} p\left(u+T_{r}\right) d u} d s \leq \lambda^{*}
$$

then we have

$$
P(t)=\underset{\xi \geq t}{\inf } p(\xi) \text { for } t \geq 0
$$

$$
\lim _{t \rightarrow \infty} \inf \int_{0}^{t} K(s) e^{r \int_{t-s}^{t} P(u) d u} d s \leq \lambda^{*}
$$

Obviously, the function $P$ is increasing on the interval $[0, \infty)$. Set

$$
F_{r}(t)=\int_{0}^{t} K(s) e^{r \int_{t-s}^{t} P(u) d u} d s \text { for } t \geq 0 .
$$

Then we obtain for every $t \geq 0$

$$
F_{r}^{\prime}(t)=\int_{0}^{t} K(s) e^{r \int_{t-s}^{t} P(u) d u} r[P(t)-P(t-s)] d s+K(t) e^{r \int_{0}^{t} P(u) d u} \geq 0
$$

and hence the function $F_{r}$ is increasing on $[0, \infty)$. So, (9) gives 


$$
\sup _{t \geq 0} \int_{0}^{t} K(s) e^{r \int_{t-s}^{t} P(u) d u} d s \leq \lambda^{*}
$$

Now, consider an arbitrary $\tau \geq 0$. From (10) it follows that

$$
\begin{aligned}
& \lambda^{*} \geq \sup _{t \geq 0} \int_{0}^{t} K(s) e^{r \int_{t-s}^{t} P(u) d u} d s \geq \int_{0}^{\tau} K(s) e^{r \int_{\tau-s}^{\tau} P(u) d u} d s \\
& =\int_{0}^{\tau} K(s) e^{\left(\lambda^{*}-\mu\right) \int_{\tau-s}^{\tau} P(u) d u} d s \geq e^{-\mu \int_{0}^{\tau} P(u) d u} \int_{0}^{\tau} K(s) e^{\lambda^{*} \int_{\tau-s}^{\tau} P(u) d u} d s
\end{aligned}
$$

and consequently

$$
\int_{0}^{\tau} K(s) e^{\lambda^{*} \int_{\tau-s}^{\tau} P(u) d u} d s \leq \lambda^{*} e^{\mu \int_{0}^{\tau} P(u) d u}
$$

Therefore, as $\mu \rightarrow 0^{+}$, we derive

$$
\int_{0}^{\tau} K(s) e^{\lambda^{*} \int_{\tau-s}^{\tau} P(u) d u} d s \leq \lambda^{*}
$$

The last inequality holds for all $\tau \geq 0$. So, we obtain

$$
\sup _{\tau \geq 0} \int_{0}^{\tau} K(s) e^{\lambda^{*} \int_{\tau-s}^{\tau} P(u) d u} d s \leq \lambda^{*}
$$

which contradicts condition $\left(C_{1}\right)$. The proof of Theorem 1 is complete.

Proof of Theorem 2: $\quad$ Assume that there is a solution $y$ on $[T, \infty)$ of $\left(I_{2}\right)$ which is positive on $R$. Set $T^{*}=\max \{0, T\} \geq 0$. Then we obtain for every $t \geq T^{*}$

$$
0 \geq y^{\prime}(t)+q(t) \int_{-\infty}^{t} K(t-s) y(s) d s \geq y^{\prime}(t)+q(t) \int_{0}^{t} K(t-s) y(s) d s
$$

So, the function $Y=y \mid[0, \infty)$ is a solution on $\left[T^{*}, \infty\right)$ of the integrodifferential inequality

$$
Y^{\prime}(t)+q(t) \int_{0}^{t} K(t-s) Y(s) d s \leq 0
$$

which is positive on $[0, \infty)$. But this is impossible, as it follows from Theorem 1 . Hence, the 
proof of Theorem 2 is complete.

Proof of Theorem 3: $\quad$ From $\left(I_{1}\right)$ we obtain for $\widehat{t} \geq t \geq 0$

and hence

$$
y(t) \geq y(\widehat{t})+\int_{t}^{\widehat{t}} p(u) \int_{0}^{u} K(u-s) y(s) d s d u>\int_{t}^{\widehat{t}} p(u) \int_{0}^{u} K(u-s) y(s) d s d u
$$

$$
y(t) \geq \int_{t}^{\infty} p(u) \int_{0}^{u} K(u-s) y(s) d s d u \text { for all } t \geq 0
$$

Let $X$ be the set of all continuous real-valued functions $x$ on the interval $[0, \infty)$ which satisfy

$$
0 \leq x(t) \leq y(t) \text { for every } t \geq 0 \text {. }
$$

Then, by using (11), we can see that the formula

$$
(S x)(t)=\left\{\begin{array}{c}
\int_{t}^{\infty} p(u) \int_{0}^{u} K(u-s) x(s) d s d u, \text { if } t \geq T \\
\int_{T}^{\infty} p(u) \int_{0}^{u} K(u-s) x(s) d s d u+\int_{t}^{T} p(u) \int_{0}^{u} K(u-s) y(s) d s d u, \text { if } 0 \leq t<T
\end{array}\right.
$$

defines an operator $S: X \rightarrow X$. This operator is increasing in the sense that, if $x_{1}, x_{2} \in X$ and $x_{1} \leq x_{2}$, then $S x_{1} \leq S x_{2}$ (here we use the usual pointwise ordering in $X$ ). Next, we set

$$
x_{0}=y, \text { and } x_{m}=S x_{m-1}(m=1,2, \ldots) \text {. }
$$

Then we immediately see that $\left(x_{m}\right)_{m=0,1, \ldots}$ is a decreasing sequence of functions in $X$. Furthermore, we define

$$
x=\lim _{m \rightarrow \infty} x_{m} \text { pointwise on }[0, \infty)
$$

By applying the Lebesgue dominated convergence theorem, we conclude that $x=S x$, i.e.

$$
x(t)=\left\{\begin{array}{c}
\int_{t}^{\infty} p(u) \int_{0}^{u} K(u-s) x(s) d s d u, \text { if } t \geq T \\
\int_{T}^{\infty} p(u) \int_{0}^{u} K(u-s) x(s) d s d u+\int_{t}^{T} p(u) \int_{0}^{u} K(u-s) y(s) d s d u, \text { if } 0 \leq t<T .
\end{array}\right.
$$

From (12) we obtain 


$$
x^{\prime}(t)=-p(t) \int_{0}^{t} K(t-s) x(s) d s \text { for all } t \geq T
$$

which means that $x$ is a solution on $[T, \infty)$ of $\left(E_{1}\right)$. Obviously, $x \leq y$ on $[0, \infty)$. Also, (12) guarantees that $x$ satisfies (1). Moreover, from (12) we derive for $0 \leq t<T$

$$
x^{\prime}(t)=-p(t) \int_{0}^{t} K(t-s) y(s) d s \leq-p(t) \int_{0}^{t} K(t-s) x(s) d s
$$

and so (2) holds. It remains to establish that $x$ is positive on the interval $[0, \infty)$. Clearly, we have for $0 \leq t<T$

$$
x(t) \geq \int_{t}^{T} p(u) \int_{0}^{u} K(u-s) y(s) d s d u=\int_{t}^{T} p(u) \int_{0}^{u} K(s) y(u-s) d s d u>0,
$$

because $p(T)>0, K$ is not identically zero on $[0, T]$ and $y$ is positive on $[0, T]$. Thus, $x$ is positive on $[0, T)$. Finally, we will show that $x$ is also positive on $[T, \infty)$. Assume, for the sake of contradiction, that $\tau \geq T$ is the first zero of $x$ to the right of 0 . That is,

$$
x(t)>0 \text { for } 0 \leq t<\tau, \text { and } x(\tau)=0 .
$$

Then (12) gives

$$
0=x(\tau)=\int_{\tau}^{\infty} p(u) \int_{0}^{u} K(u-s) x(s) d s d u=\int_{\tau}^{\infty} p(u) \int_{0}^{u} K(s) x(u-s) d s d u
$$

and therefore

$$
p(u) \int_{0}^{u} K(s) x(u-s) d s=0 \text { for all } u \geq \tau
$$

In particular, we have

$$
p(\tau) \int_{0}^{\tau} K(s) x(\tau-s) d s=0
$$

This is impossible, because $p(\tau)>0, K$ is not identically zero on $[0, T]$ and $x$ is positive on $[0, \tau)$. The proof is complete.

Proof of Corollary 1: Set

$$
y(t)=e^{-\lambda \int_{0}^{t} p(u) d u} \text { for } t \geq 0 .
$$

Then we obtain for every $t \geq 0$ 


$$
\begin{aligned}
& y^{\prime}(t)+p(t) \int_{0}^{t} K(t-s) y(s) d s=y^{\prime}(t)+p(t) \int_{0}^{t} K(s) y(t-s) d s \\
& =p(t) e^{-\lambda \int_{0}^{t} p(u) d u}\left[-\lambda+\int_{0}^{t} K(s) e^{\lambda \int_{t-s}^{t} p(u) d u} d s\right] \\
& \leq p(t) e^{-\lambda \int_{0}^{t} p(u) d u}\left[-\lambda+\sup _{t \geq 0} \int_{0}^{t} K(s) e^{\lambda \int_{t-s}^{t} p(u) d u} d s\right] .
\end{aligned}
$$

Hence, by hypothesis $\left(H_{1}\right)$, the function $y$ is a positive solution on $[0, \infty)$ of $\left(I_{1}\right)$ and so it suffices to apply Theorem 3 .

Proof of Theorem 4: $\quad$ From $\left(I_{2}\right)$ it follows that

$$
y(t) \geq \int_{t}^{\infty} q(u) \int_{-\infty}^{u} K(u-s) y(s) d s d u \text { for } t \in R .
$$

Consider the set $X$ of all nonnegative continuous functions on $R$ with $x(t) \leq y(t)$ for every $t \in R$. Then, in view of (13), the formula

$$
(S x)(t)=\left\{\begin{array}{c}
\int_{t}^{\infty} q(u) \int_{-\infty}^{u} K(u-s) x(s) d s d u, \text { if } t \geq T \\
\int_{T}^{\infty} q(u) \int_{-\infty}^{u} K(u-s) x(s) d s d u+\int_{t}^{T} q(u) \int_{-\infty}^{u} K(u-s) y(s) d s d u, \text { if } t<T
\end{array}\right.
$$

defines an increasing mapping $S$ of $X$ into itself. Define the decreasing sequence $\left(x_{m}\right)_{m}=0,1, \ldots$ of functions in $X$, where

$$
x_{0}=y \text { and } x_{m}=S x_{m-1} \quad(m=1,2, \ldots)
$$

Furthermore, set

$$
x=\lim _{m \rightarrow \infty} x_{m} \text { pointwise on } R .
$$

By the Lebesgue dominated convergence theorem, we obtain $x=S x$. That is,

$$
x(t)=\left\{\begin{array}{c}
\int_{t}^{\infty} q(u) \int_{-\infty}^{u} K(u-s) x(s) d s d u, \text { if } t \geq T \\
\int_{T}^{\infty} q(u) \int_{-\infty}^{u} K(u-s) x(s) d s d u+\int_{t}^{T} q(u) \int_{-\infty}^{u} K(u-s) y(s) d s d u, \text { if } t<T .
\end{array}\right.
$$


Then

$$
x^{\prime}(t)=-q(t) \int_{-\infty}^{t} K(t-s) x(s) d s \text { for } t \geq T
$$

and consequently $x$ is a solution on $[T, \infty)$ of $\left(E_{2}\right)$. This solution is such that $x(t) \leq y(t)$ for $t \in R$. Also, because of (14), $x$ satisfies (1). Moreover, from (14) we obtain for $t<T$

$$
x^{\prime}(t)=-q(t) \int_{-\infty}^{t} K(t-s) y(s) d s \leq-q(t) \int_{-\infty}^{t} K(t-s) x(s) d s
$$

and hence (3) holds. Finally, we will show that $x$ is positive on $R$. Since $q(T)>0, y$ is positive on $R$ and $K$ is not identically zero on $[0, \infty)$, we have

and so

$$
q(T) \int_{-\infty}^{T} K(T-s) y(s) d s>0
$$

$$
\int_{t}^{T} q(u) \int_{-\infty}^{u} K(u-s) y(s) d s d u>0, \text { if } t<T
$$

Hence, because of (14), the solution $x$ is positive on $(-\infty, T)$. Next, we shall prove that $x$ is also positive on $[T, \infty)$. Let $\tau \geq T$ be the first zero of $x$. Then $x(\tau)=0$ and $x(t)>0$ for $t<\tau$. We have, by (14),

and consequently

$$
0=x(\tau)=\int_{\tau}^{\infty} q(u) \int_{-\infty}^{u} K(u-s) x(s) d s d u
$$

In particular, we obtain

$$
q(u) \int_{-\infty}^{u} K(u-s) x(s) d s=0 \text { for all } u \geq \tau
$$

$$
q(\tau) \int_{-\infty}^{\tau} K(\tau-s) x(s) d s=0
$$

which contradicts the fact that $q(\tau)>0, K$ is not identically zero on $[0, \infty)$ and $x$ is positive on $(-\infty, \tau)$. The proof is now complete.

Proof of Corollary 2: $\quad$ Define

$$
y(t)=e^{-\lambda \int_{0}^{t} q(u) d u} \text { for } t \in R
$$

Then, for every $t \in R$, we get

$$
y^{\prime}(t)+q(t) \int_{-\infty}^{t} K(t-s) y(s) d s=y^{\prime}(t)+q(t) \int_{0}^{\infty} K(s) y(t-s) d s
$$




$$
\begin{gathered}
=q(t) e^{-\lambda \int_{0}^{t} q(u) d u}\left[-\lambda+\int_{0}^{\infty} K(s) e^{\lambda \int_{-s}^{t} q(u) d s}\right] \\
\leq q(t) e^{-\lambda \int_{0}^{t} q(u) d u}\left[-\lambda+\sup _{t \in R} \int_{0}^{\infty} K(s) e^{\lambda \int^{t} q(u) d u} d s\right] .
\end{gathered}
$$

Now, condition $\left(\mathrm{H}_{2}\right)$ guarantees that $y$ is a positive solution on $R$ of $\left(I_{2}\right)$. So, it is enough to apply Theorem 4 .

\section{REFERENCES}

[1] Burton, T.A., Volterra Integral and Differential Equations, Academic Press, New York 1983.

[2] Corduneanu, C. and Lakshmikantham, V., Equations with unbounded delay: A survey, Nonlinear Analysis 4, (1980), pp. 831-877.

[3] Gopalsamy, K., Stability, instability, oscillation and nonoscillation in scalar integrodifferential systems, Bull. Austral. Math. Soc. 28, (1983), pp. 233-246.

[4] Gopalsamy, K., Non-oscillation in a delay-logistic equation, Quart. Appl. Math. 43, (1985), pp. 189-197.

[5] Gopalsamy, K., Oscillations in a delay-logistic equation, Quart. Appl. Math. 44, (1986), pp. 447-461.

[6] Gopalsamy, K., Oscillations in systems of integrodifferential equations, J. Math. Anal. Appl. 113, (1986), pp. 78-87.

[7] Gopalsamy, K., Oscillations in integrodifferential equations of arbitrary order, $J$. Math. Anal. Appl. 126, (1987), pp. 100-109.

[8] Gopalsamy, K., Stability and nonoscillation in a logistic equation with recruitment delays, Nonlinear Anal. 11, (1987), pp. 199-206.

[9] Ladas, G., Philos, Ch.G. and Sficas, Y.G., Oscillations of integrodifferential equations, Differential and Integral Equations 4, (1991), pp. 1113-1120.

[10] Philos, Ch.G., Oscillatory behavior of systems of integrodifferential equations, Bull. Soc. Math. Grèce (N.S.) 29, (1988), pp. 131-141.

[11] Philos, Ch.G., Oscillation and nonoscillation in integrodifferential equations, Libertas Math. (to appear).

[12] Philos, Ch.G. and Sficas, Y.G., On the existence of positive solutions of integrodifferential equations, Applicable Anal. 36, (1990), pp. 189-210. 


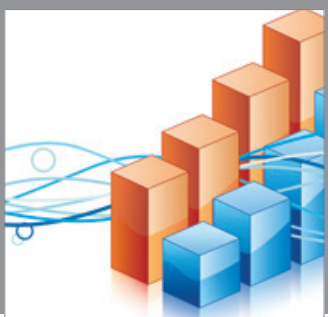

Advances in

Operations Research

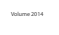

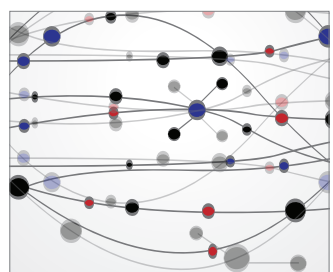

\section{The Scientific} World Journal
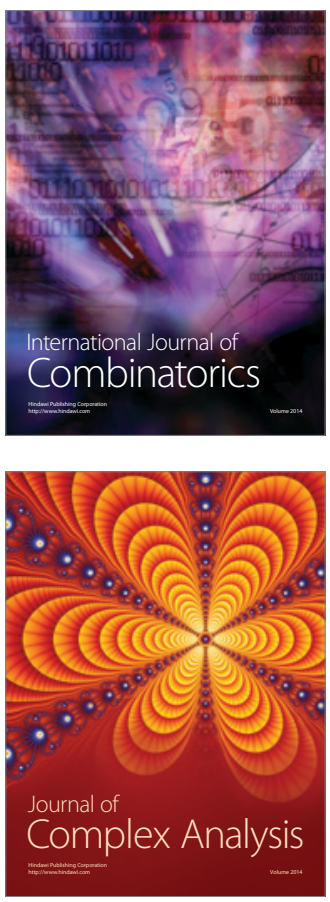

International Journal of

Mathematics and

Mathematical

Sciences
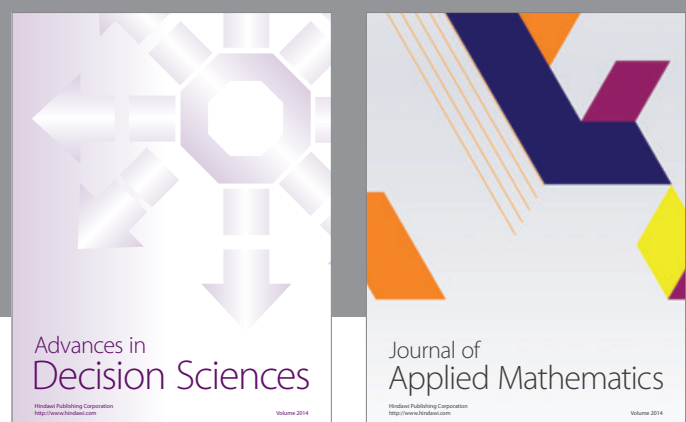

Journal of

Applied Mathematics
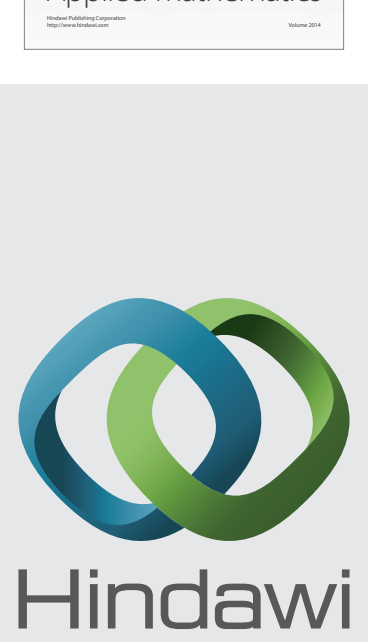

Submit your manuscripts at http://www.hindawi.com
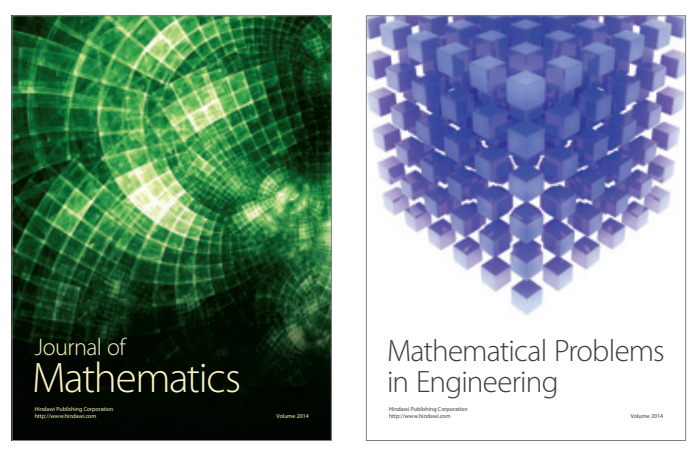

Mathematical Problems in Engineering
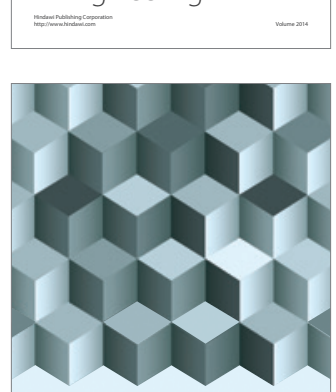

Journal of

Function Spaces
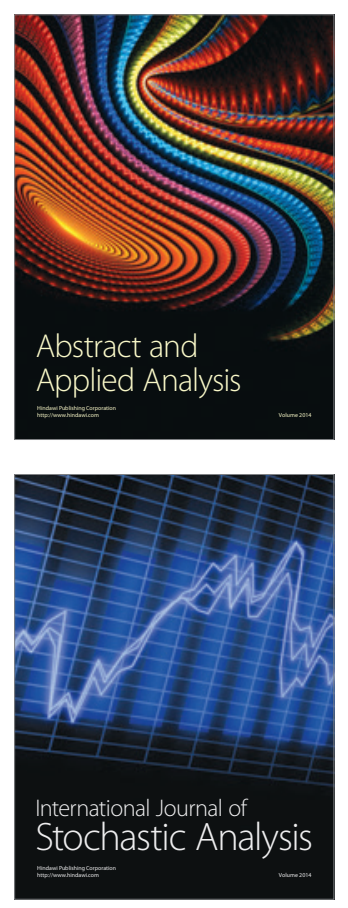

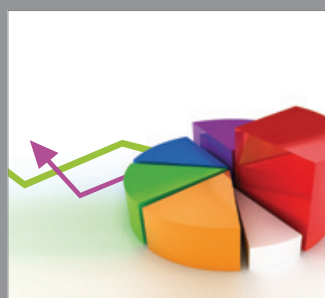

ournal of

Probability and Statistics

Promensencen
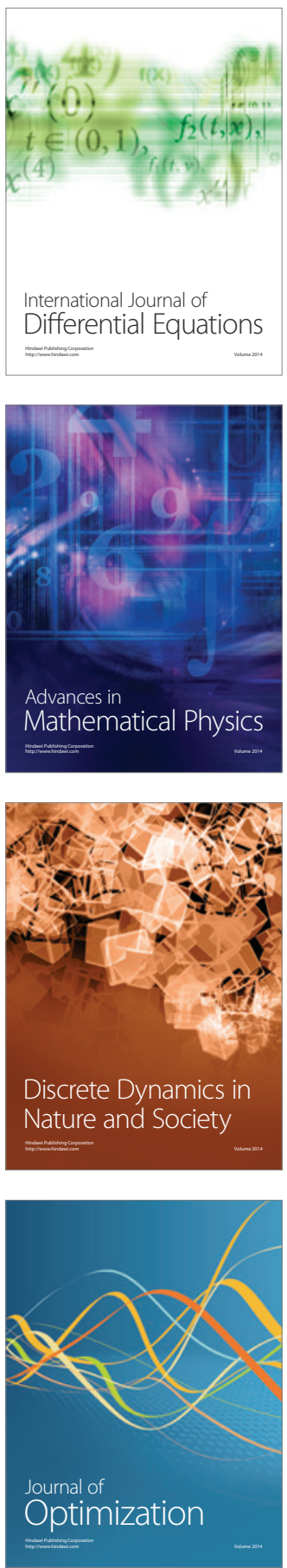